\title{
Experimental Study of the Dominant Flow Paths and Analysis of the Influence Factors Through Fracture Networks
}

\author{
Mu Wang ${ }^{1,2, ~ *, ~ F e n g j u n ~ G a o ², ~ J i a z h o n g ~ Q i a n ~}{ }^{2}$ \\ ${ }^{1}$ Anhui Guozhen Environmental Restoration Company Limited by Shares, Hefei, China \\ ${ }^{2}$ School of Resources and Environmental Engineering, Hefei University of Technology, Hefei, China \\ Email address: \\ wangmucjl@163.com (Mu Wang),1239631555@qq.com (Fengjun Gao), qianjiazhong@hfut.edu.cn(Jiazhong Qian) \\ ${ }^{*}$ Corresponding author
}

To cite this article:

Mu Wang, Fengjun Gao, Jiazhong Qian. Experimental Study of the Dominant Flow Paths and Analysis of the Influence Factors Through Fracture Networks. Hydrology. Vol. 7, No. 2, 2019, pp. 32-37. doi: 10.11648/j.hyd.20190702.12

Received: July 11, 2019; Accepted: August 12, 2019; Published: August 26, 2019

\begin{abstract}
As the main carrier of groundwater in nature, water transport behavior in fracture networks and identification of its main control factors are challenging problems in hydrogeology. Laboratory experiments are designed in this paper using fracture networks made of Perspex plate for a series of hydraulic tests. Using the conditions of different types of connections for inlet and outlet, temperature tracing tests are conducted for determining dominant flow paths. The flow resistances are calculated at different points, and the control factors of the dominant flow paths are then discussed. Three major conclusions are obtained: (1) the existence of the dominant flow phenomenon in fracture networks is verified; (2) the dominant flow paths can be ascertained by monitoring the temperature variation of hot water in complex fracture networks; (3) the flow resistance is the most fundamental reason for forming dominant flow: the channel with less resistance is selected as the dominant path.
\end{abstract}

Keywords: Dominant Flow Paths, Temperature Tracer, Flow Resistance, Fracture Networks

\section{Introduction}

Fracture formations are ubiquitous in nature and are related to a number of geological activities including the exploitation of shale gas. Fracture formations may occur in the form of a single fracture or in a fracture network, in which the fracture network is the common ingredient, making the study of water flow in fracture networks an attractive topic. Cherubini et al. [1] investigated non-linear flow by analyzing hydraulic tests on an artificially-created fractured rock sample, and the result showed that the relationship between the flow rate discharge and the head gradient characterized a strong inertial regime. Zhang et al [2] investigated the fluid flow regimes through deformable rock fractures by conducting water flow tests through both mated and non-mated sandstone fractures. Quinn et al. [3] quantified the non-Darcian flow by packer testing. They found that the flow is non-linear but not quadratic in nature. Chen et al. [4] conducted test and simulation for the fracture network dominant flow, the results showing that under the same hydraulic conditions, water flowing through a fracture network made an earlier transition to a turbulent state than through a single fracture, by the effect of fracture intersection.

Determination of flow paths is the foundational research of fracture networks: the dominant flow is formed where the flow is concentrated. Berkowitz [5] summarized the flow and solute transport laws of heterogeneous fractured rock mass, and pointed out that the dominant flow path in fracture networks was the most important factor affecting fracture flow. Salve [6] conducted field tests to study the dominant flow and formation mechanisms in different rock fractures. The study shown that the number of fracture seepage channels in the rock mass only accounted for $10 \%$ to $20 \%$ of the total, the remaining fractures were mostly disconnected or poorly conductive. Figueiredo et al. [7] carried out both laboratory and field tests, since the experimental results of water flow and solute transport in the conventional sealed fracture network model may be inaccurate when compared to the actual situation. In the sparse channel, penetration was dominated by the shape of the fracture, and the size and direction of the fracture had less impact. Domestic scholar, Tian [8] found the phenomenon of fracture water bias 
flow by studying unequal cross fractures and explained the formation mechanism of fracture water runoff. Yang et al. [9] designed the physical model of cross-fracture with different aspects of ratio and roughness, and simulated the experimental data by using Fluent software, and studied the non-Darcy bias effect characteristics of the cross-rough fracture under the condition of high flow rate.

However, it is difficult to recognize dominant flow paths given the complexity and high numbers of channels. Seepage characteristics have been the subject of several studies monitoring temperature data in fracture media. Rau et al. [10] proposed that temperature could be used to monitor groundwater seepage exchange. Temperature was widely used as a tracer in the study of groundwater after the development of the waterproof temperature recorder. Becker [11] used airborne temperature sensors to study regional groundwater flow. Silliman and Robinson [12] estimated the position of fracture with the change of temperature, the contamination of limestone fracture aquifer from surface sewage infiltration was studied by using a temperature tracer. Klepikova et al. [13] compared the different test methods based on the principle of dominant flow, and proposed that temperature tomography seemed to be an effective method to detect the dominant flow path and characterize its hydraulic properties. Cherubini et al. [14] designed experimental tests on heat transport in the fracture network, and simulated temperature curves with a particular network model, the results showing that heat transport appeared to delay the effect compared with solute transport. In this study, temperature data is calculated as a tracer for reflecting the dominant flow paths.

Many scholars have used different methods to analyze numerous control factors for forming the dominant flow paths, such as fracture connectivity, geometric characteristics and degree of filling. Tian [15] proposed three important hydraulic characteristics for the theory of fracture water bias flow. One was bias flow, another, deflection, and the third was the law of resistance inequality, all significant reasons for the formation of dominant flow in fractured media. Gong and Rossen [16] focused on the influence of fracture aperture distribution, and found that even a well-connected fracture network could behave like a much sparser network when the aperture distribution was broad enough. Chuang et al. [17] used nanoscale zero-valent iron (nZVI) particles as tracers to characterize fracture connectivity between two boreholes in fractured rock. The position where the maximum weight of attracted nZVI particles was observed coincides with the depth of a permeable fracture zone delineated by the heat-pulse flowmeter. Somogyvári et al. [18] presented a novel concept by using a transdimensional inversion method to invert a two-dimensional cross-well discrete fracture network (DFN) geometry from tracer tomography experiments. The procedure successfully identified major transport pathways in the investigated domain and explored equally probable DFN realizations, which were analyzed in fracture probability maps and by multidimensional scaling.

To sum up, there are few articles about analyzing the influence of flow resistance to dominant flow paths through fracture networks. Therefore, this issue is discussed by method of laboratory experiments. The purposes of this study are to: (1) verify the existence of dominant flow phenomenon in fracture networks; (2) ascertain the dominant flow paths of different fracture connection types; and (3) analyze the influence factors to dominant flow paths.

\section{Basic Equations}

From the perspective of fluid mechanics, the reason for the dominant flow in the fracture networks is that the water flows in different channels affected by different resistances. The large flux, the less resistance. In the calculation process of project, the energy loss is divided into two categories according to whether the side wall of the fluid contact changes along the path: energy loss along the path and local energy loss. When the boundary of the channel is nearly unchanged along the path, the flow resistance is also unchanged basically, which is called the frictional resistance. The head loss caused by the frictional resistance can be expressed as:

$$
\mathrm{h}_{\mathrm{f}}=\lambda \frac{1}{2 \mathrm{~b}} \frac{\mathrm{v}^{2}}{2 \mathrm{~g}}
$$

where $l$ is the fracture length, $b$ is the aperture, $v$ is the average velocity, and $\lambda$ is the resistance coefficient, it can be calculated by:

$$
\lambda=0.11\left(\frac{68}{R_{e}}\right)^{0.25}
$$

where $R e$ is the Reynolds number of corresponding velocity, it is expressed as:

$$
\mathrm{R}_{\mathrm{e}}=\frac{\mathrm{vb}}{v}
$$

where $v$ is the kinematic viscosity of the fluid (in this experiment, the value of kinematic viscosity is $1.2028 \times 10^{-6} \mathrm{~m}^{2} / \mathrm{s}$ ).

When the fluid flows through various local obstacles, the uniform flow is destroyed in this local area due to the change of flux or boundary, causing the change of the distribution, size or direction of the flow velocity. This resistance brings energy loss in the pipeline and is called local resistance. The head loss caused by the local resistance can be expressed as:

$$
\mathrm{h}_{\mathrm{m}}=\xi \frac{\mathrm{v}^{2}}{2 \mathrm{~g}}
$$

where $\xi$ is the local resistance coefficient, the corresponding values of $\xi$ under conditions of different cross angles can be

\begin{tabular}{|c|c|c|c|c|c|c|c|c|c|c|c|}
\hline cross angle & $\mathbf{0}$ & 10 & 20 & 30 & 40 & 50 & 60 & 70 & 75 & 80 & 85 \\
\hline$\xi$ & 0.50 & 0.56 & 0.63 & 0.70 & 0.78 & 0.85 & 0.91 & 0.96 & 0.98 & 0.99 & 1.00 \\
\hline
\end{tabular}
found in the common data of the water supply and drainage design manual, as shown in Table 1.

Table 1. Statistics of local resistance coefficient of cross fracture. 
In the fracture networks, the water flow resistance needs to consider both the frictional resistance and the local resistance, then the head loss of each channel is expressed as:

$$
\mathrm{h}=h_{f}+h_{m}
$$

\section{Experimental Design}

\subsection{Experimental Setup}

The schematic diagram of the experimental setup for groundwater flow tests in a fracture networks is shown in Figure 1. The model is circular and made with $0.600 \mathrm{~m}$ diameter Perspex casing, consisting of three major parts: inflow (outflow) flumes, fiber optic temperature sensor, and the fracture network. A fracture network is formed in the 60-degree sections, divided by two main fractures with an aperture of $0.008 \mathrm{~m}$ (horizontal fracture) and $0.006 \mathrm{~m}$. The two sections are of equal size and the center of symmetry at the center point and the model is sealed with a Perspex plate cover. Each region is filled with cuboid plates with dimensions of $0.030 \mathrm{~m} \times 0.030 \mathrm{~m} \times 0.005 \mathrm{~m}$ (length $\times$ width $\times$ height $)$ spaced at $0.003 \mathrm{~m}$. Three groups of inlets and outlets are designed at the edge of the model marked as 1-6 respectively. Holes are drilled along each horizontal fracture for inserting the temperature sensor probes which connect with NZS-FBG-A01 (D) fiber optic demodulator, with uneven numbers of monitoring points distribute on each probe with a spacing of $0.033 \mathrm{~m}$. Locations are shown with blue points in Figure 1 (b). The flow tests are conducted by changing the connection type of inlet and outlet and the water velocity.

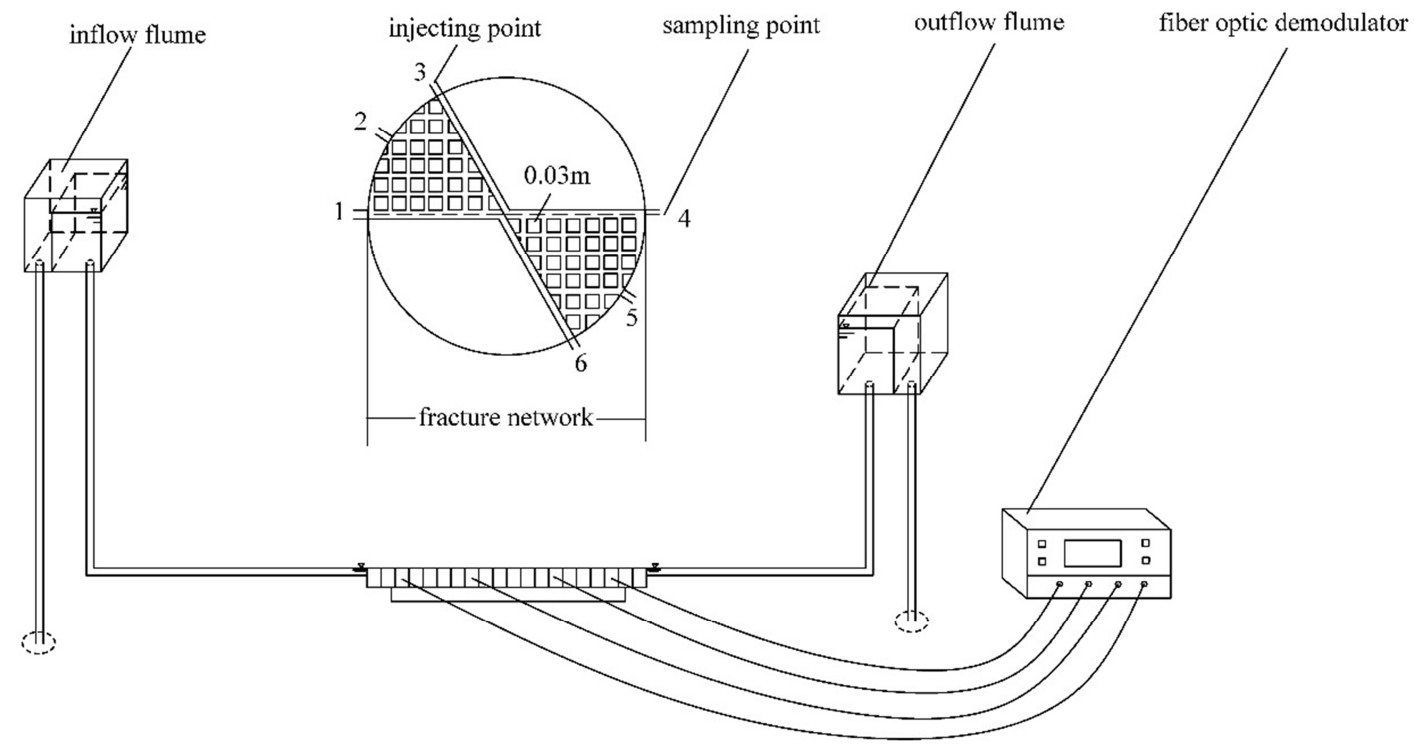

(a)

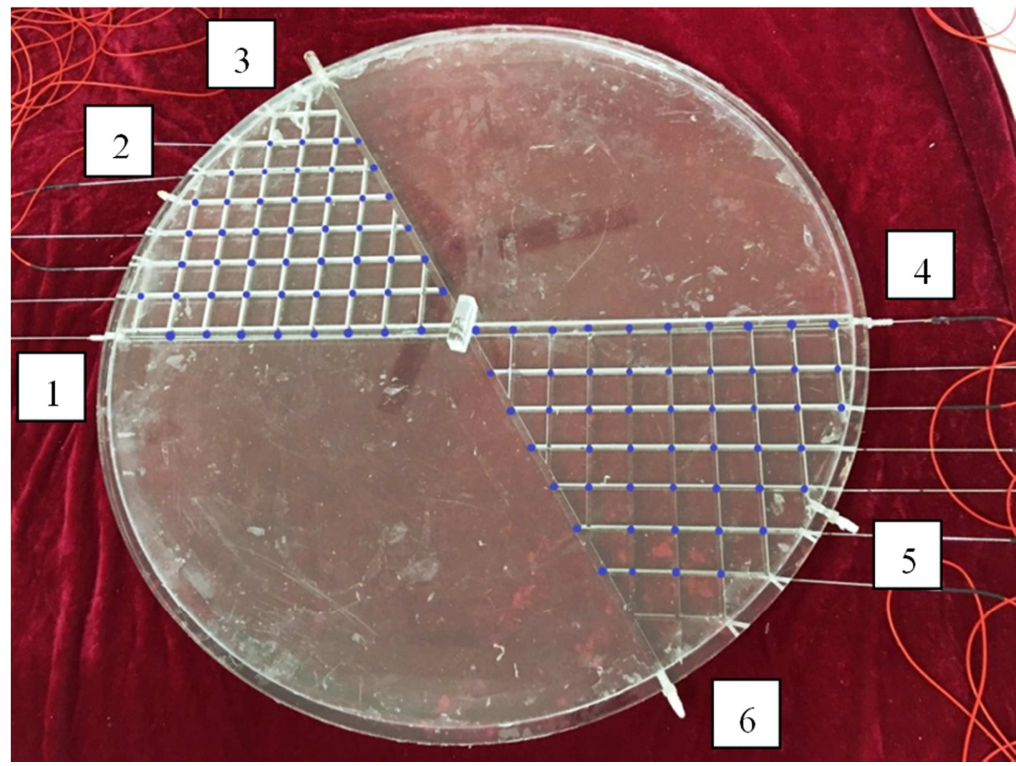

(b)

Figure 1. Schematic diagrams of experimental setup: (a) experimental whole model, (b) circular fracture network. 


\subsection{Testing Method}

The cylinder is used to measure the steady flow rate, with an error rate less than $2 \%$. The water level is measured by piezometric tubes with an error of $\pm 0.0005 \mathrm{~m}$. The value of temperature variation is equal to the temperature coefficient multiplied by the value of the wavelength variation, which is monitored by the probe with an error of \pm 0.1 degree.

\section{Experimental Results and Discussions}

There are four connection types in the circular fracture network: inlet 2 -outlet 5 , inlet 2 -outlet 6 , inlet 3 -outlet 5 , and inlet 3 -outlet 6 . The original experimental data includes the water head, flux and temperature data (calculate from wavelength values) in the fracture network.

\subsection{Verification the Existence of Dominant Flow Phenomenon}

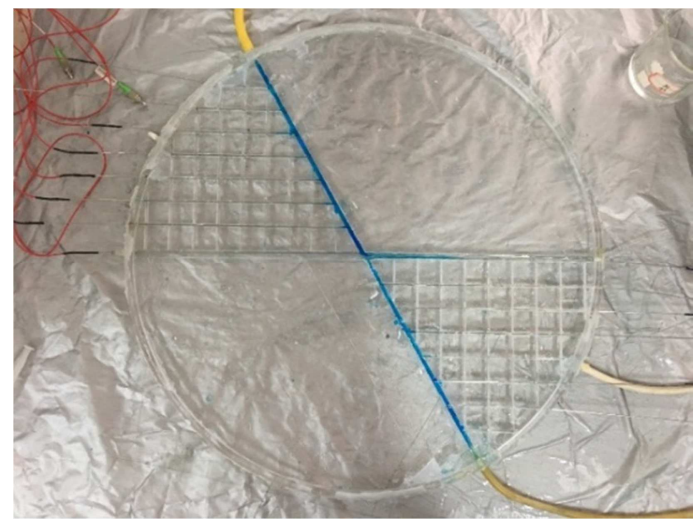

(a)

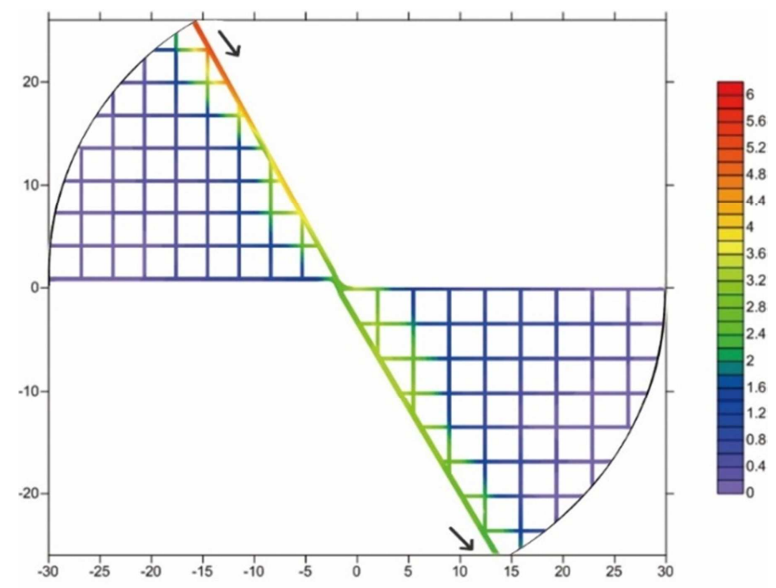

(b)

Figure 2. Schematic diagram of dominant flow path of inlet 3-outlet 6 in circular fracture networks: (a) brilliant blue transport path, (b) diagram of temperature variation.
During every test, $10 \mathrm{~mL}$ of hot water is injected into the channels, the wavelengths of water are monitored under the condition of different velocities then the temperature values of different monitoring points are calculated. For example, under the condition of inlet 3 -outlet 6 , hot water is injected with brilliant blue. The initial transport path of brilliant blue is shown in Figure 2 (a), where the main fracture of inlet 3 -outlet 6 is the dominant flow path obviously. It is found that the value of the temperature variation at the monitoring points in this main fracture is at its maximum in each probe.

From here, the boundary condition of the circular fracture networks is constructed according to the values of temperature variation monitored by each probe point. The interpolation algorithm is used in Surfer ${ }^{\circledR}$ to generate grid files for obtaining the temperature distribution diagram covering the entire model area. Different colors are used to indicate the temperature levels, and the dominant flow path is structured by connecting the maximum values, the result is shown in Figure 2 (b). The existence of the dominant flow phenomenon in the network crack is thus confirmed, and it is reasonable to connect the monitoring points with the maximum values of temperature variation for forming the dominant flow paths.

\subsection{Ascertaining the Dominant Flow Paths of Fracture Network}

In order to display the dominant flow paths more clearly, three-dimensional histograms are drawn using Python software based on the temperature monitoring data. The column heights and different colors are used to reflect the temperature variation of each probe, and dominant flow paths are constituted by connecting them. Taking the flow rate of $0.002 \mathrm{~m} / \mathrm{s}$ and the hot water injection amount of $20 \mathrm{~mL}$ as an example, the monitoring of temperature distribution obtained at $24 \mathrm{~s}$ and the corresponding dominant flow paths are shown in Figure 3.

In Figure 3 (a), hot water is injected into inlet 2, the peak points of temperature variation distribute along network channels into the main fracture with aperture of $0.008 \mathrm{~m}$, then circuitously leads to outlet 5 . In Figure 3 (c), the distribution of the peak points is initially similar to Figure 3 (a), but then directly to outlet 6 . In Figure 3 (e) and 3 (g), hot water is injected into inlet 3 , the peak points distribute in the main fracture with an aperture of $0.006 \mathrm{~m}$, then meander to outlet 5 and directly leads to outlet 6 , respectively. The Figure 3 (b), (d), (f), (h) are the dominant flow paths drawn according to the figures of temperature variation on the left side, respectively. 


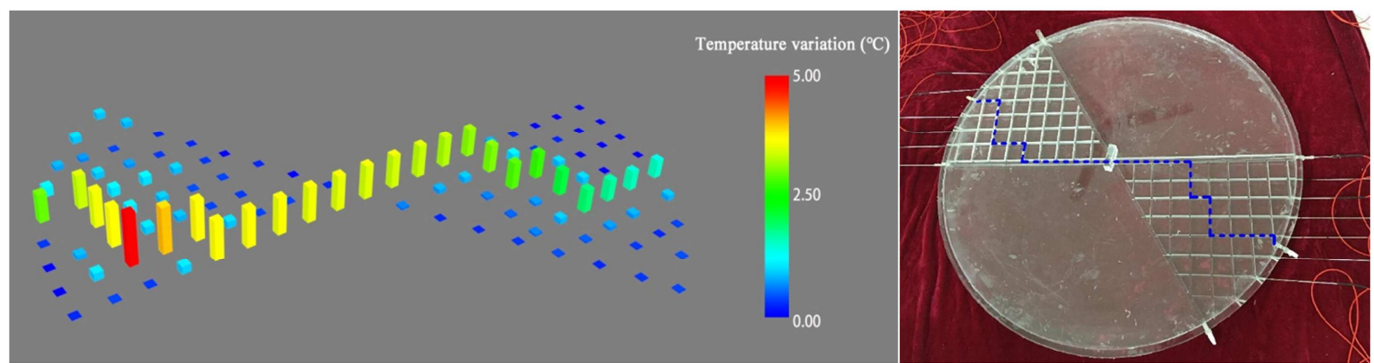

(a)

(b)

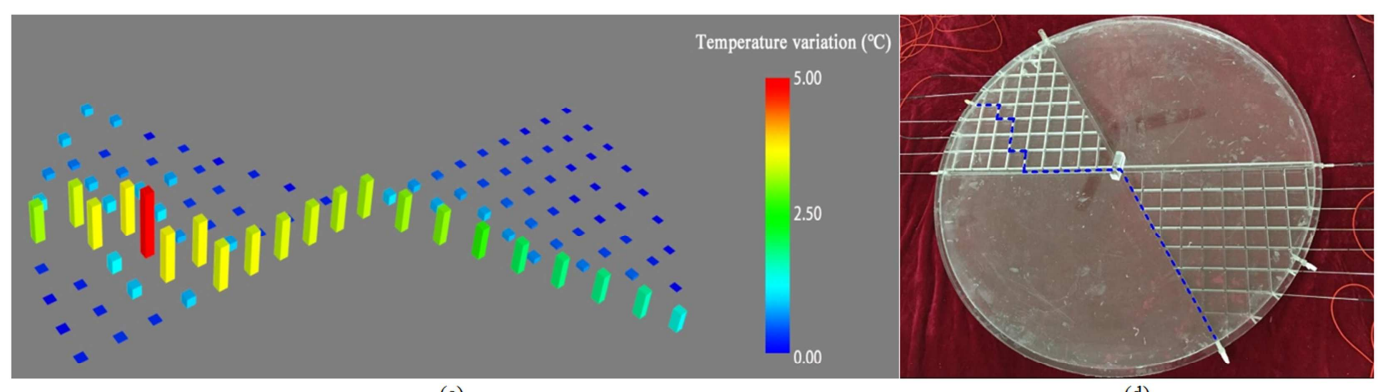

(c)

(d)

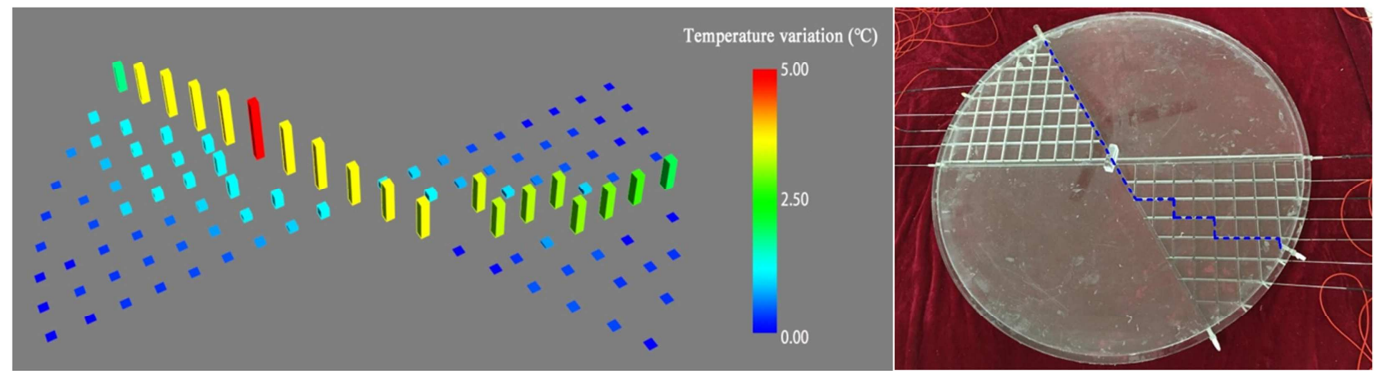

(e)

(f)

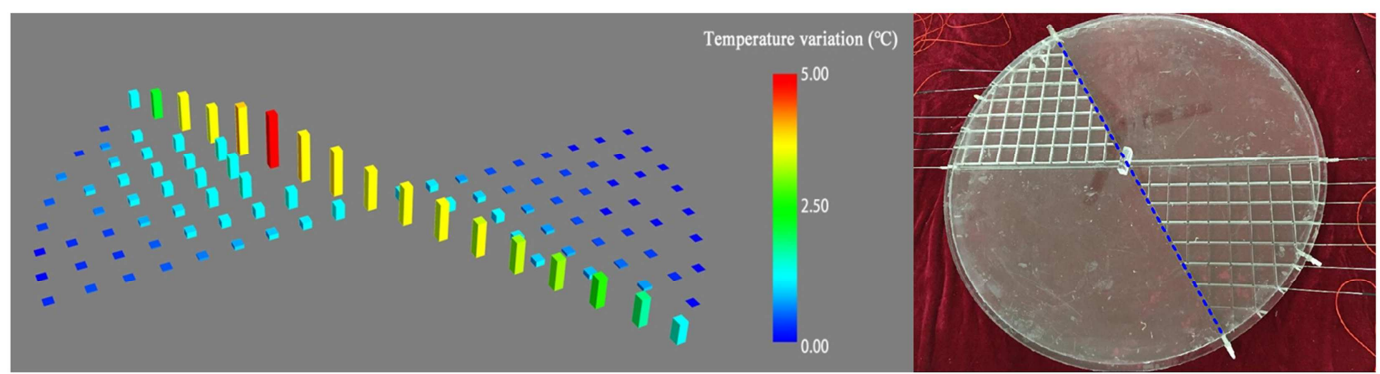

(g)

(h)

Figure 3. Temperature variation and dominant flow paths in circular fracture networks: (a) and (b) inlet 2-outlet 5, (c) and (d) inlet 2-outlet 6, (e) and (f) inlet 3-outlet 5, $(\mathrm{g})$ and $(\mathrm{h})$ inlet 3-outlet 6.

\subsection{Analysis the Influence Factors to Dominant Flow Paths}

From the analysis above, water flow in different channels is affected by different resistances in the fracture networks. There is little resistance with a fast flow rate, and this forms the dominant flow paths. Taking inlet 2-outlet 5 as an example, the first influence factor of direction from inlet to outlet is marked, and the corresponding head losses of possible channels are expressed as $h_{1}$ and $h_{2}$, they are shown in Figure 4. The specific path is controlled by the flow resistance, this is the second and most important influence factor. The third influence factor is the main fracture with aperture of $0.008 \mathrm{~m}$ for the increasing of aperture. The values of $h_{1}$ and $h_{2}$ are calculated by equation 5 under the condition of different flow velocities, the results are shown in Table 2.

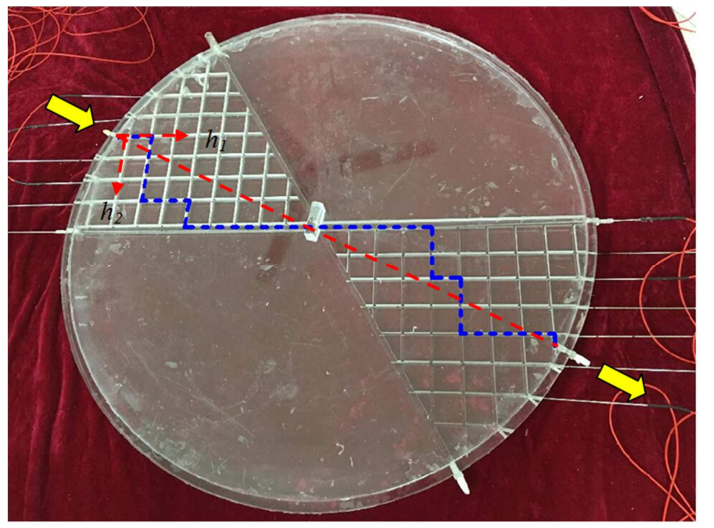

Figure 4. Diagram analysis the influence factors to dominant flow paths. 
Table 2. Statistics of flow resistance of different channels in circular fracture networks.

\begin{tabular}{lll}
\hline Flow velocities $\left(\mathbf{m} / \mathbf{s},{ }^{\times} \mathbf{1 0}^{-3}\right)$ & Values of $\boldsymbol{h}_{\boldsymbol{1}}\left(\mathbf{m},{ }^{\times} \mathbf{1 0}^{-3}\right)$ & Values of $\boldsymbol{h}_{\mathbf{2}}\left(\mathbf{m},{ }^{\times} \mathbf{1 0}{ }^{-3}\right)$ \\
\hline 0.95 & 1.50 & 10.07 \\
1.28 & 2.12 & 13.48 \\
1.64 & 2.52 & 17.62 \\
1.77 & 3.03 & 20.14 \\
1.95 & 3.54 & 23.15 \\
2.24 & 4.62 & 27.66 \\
2.46 & 5.37 & 31.72 \\
2.77 & 6.56 & 40.33 \\
3.15 & 7.64 & 50.26 \\
\hline
\end{tabular}

The values of $h_{1}$ are all smaller than that of $h_{2}$ in Table 2 , it means the flow resistance of horizontal channel is less than the vertical channel, and their difference increases with the increase of flow velocity, the channel with less resistance is selected by water, and then dominant flow paths of horizontal direction are formed in Figure 4. On the vertical direction, flow are influenced by the main fracture with aperture of $0.008 \mathrm{~m}$ more than other factors, about how to quantitatively analyze this attraction, further study is needed.

\section{Conclusions}

A model of fracture networks including of different inlets and outlets is designed in this paper, a series of hydraulic tests and temperature tracing tests are conducted under different conditions. From the analysis above, the following conclusions are drawn:

Firstly, water doesn't flow uniformly in the fracture networks, the phenomenon of dominant flow is existed and it can be verified through brilliant blue tracing tests. Secondly, it is difficult to monitor the values of flow velocities continuously in the channels of fracture networks, then the dominant flow paths can be determined by monitoring the values of temperature variation because water flow is a carrier of temperature. Lastly, the dominant flow paths are influenced by many factors including the flow direction from inlet to outlet, the flow resistance, and the aperture variation of fracture channels. The flow resistance is the most fundamental reason among them, flow finally chooses the channel with the least resistance under the action of various factors.

\section{Acknowledgements}

This work was supported by the National Natural Science Foundation of China: Experiment and Simulation of Groundwater Selective Flow in Fracture networks (Grant No. 41772250).

\section{References}

[1] Cherubini C., Giasi C. I., and Pastore N. Bench scale laboratory tests to analyze non-linear flow in fractured media [J]. Hydrology and Earth System Sciences, 2012, 16 (8): 2511-2522.

[2] Zhang Z., Nemcik J. Fluid flow regimes and nonlinear flow characteristics in deformable rock fractures [J]. Journal of Hydrology, 2013, 477: 139-151.

[3] Quinn P. M., Cherry J. A., and Parker B. L. Quantification of non-Darcian flow Observed during packer testing in fractured sedimentary rock [J]. Water Resources Research, 2011, 47 (9): W09533.

[4] Chen X. B., Zhao J., and Chen L. Experimental and Numerical Investigation of Preferential Flow in Fractured Network with Clogging Process $[\mathrm{J}]$. Mathematical Problems in Engineering, 2014, 2014 (3): 1-13.

[5] Berkowitz B. Characterizing flow and transport in fractured geological media: A review [J]. Advances in Water Resources, 2002, 25 (8): 861-884.

[6] Salve R. Observations of preferential flow during a liquid release experiment in fractured welded tuffs [J]. Water Resources Research, 2005, 41 (9): 477-487.

[7] Figueiredo B., Tsang C. F., Niemi A., et al. Review: The state-of-art of sparse channel models and their applicability to performance assessment of radioactive waste repositories in fractured crystalline formations [J]. Hydrogeology Journal, 2016, 24 (7): 1-16.

[8] Tian K. M. Bias flow and vein fracture water runoff [J]. Geological Review, 1983, 29 (5): 408-417.

[9] Yang H. H., Wang Y., Gao W., Niu Y. L. Effect of wide-gap ratio and roughness on high-speed non-Darcy bias flow effect of cross-fracture $[\mathrm{J}]$. Science Technology and Engineering, 2018, 18 (7): 44-49.

[10] Rau G. C., Andersen M. S., Mccallum A. M., et al. Analytical methods that use natural heat as a tracer to quantify surface water-groundwater exchange, evaluated using field temperature records [J]. Hydrogeology Journal, 2010, 18 (5): 1093-1110.

[11] Becker M. W. Potential for Satellite Remote Sensing of Ground Water [J]. Groundwater, 2006, 44 (2): 306-318.

[12] Silliman S., Robinson R. Identifying fracture interconnections between boreholes using natural temperature profiling: I. Conceptual Basis [J]. Groundwater, 1989, 27 (3): 393-402.

[13] Klepikova M. V., Borgne T. L., Bour O., et al. Passive temperature tomography experiments to characterize transmissivity and connectivity of preferential flow paths in fractured media $[\mathrm{J}]$. Journal of Hydrology, 2014, 512 (9): 549-562.

[14] Cherubini C., Pastore N., Giasi C. I., et al. Laboratory experimental investigation of heat transport in fractured media [J]. Nonlinear Processes in Geophysics, 2017, 24 (1): 1-37.

[15] Tian K. M.. The hydraulic properties of crossing-flow in an intersected fracture $[J]$. Acta Geological Sinica, 1986 (2): 90-102.

[16] Gong J., Rossen W. R. Modeling flow in naturally fractured reservoirs: effect of fracture aperture distribution on dominant sub-network for flow [J]. Petroleum Science, 2017, 14 (1): 138-154.

[17] Chuang P. Y., Chia Y., Chiu Y. C., et al. Mapping fracture flow paths with a nanoscale zero-valent iron tracer test and a flowmeter test [J]. Hydrogeology Journal, 2017, 26 (1): 321-331.

[18] Somogyvári M., Jalali M., Jimenez P. S., et al. Synthetic fracture networks characterization with transdimensional inversion [J]. Water Resources Research, 2017, 53 (6): 5104-5123. 